\title{
Chapter 23 \\ Thermodynamics of Hot Nuclear Matter: 1978 in the Statistical Bootstrap Model
}

\author{
Johann Rafelski and Rolf Hagedorn
}

\begin{abstract}
We formulate the statistical bootstrap model for nuclear matter, and study its resulting thermodynamic properties at nuclear densities below the saturation density. We discuss the relevance of limiting temperature and the phase transition gas-'liquid' when the volume of the fireball grows with its energy.
\end{abstract}

Editor's comment: The numerical results shown are obtained neglecting antibaryons. In obtaining these results we did find that as temperature rises this effect causes an unphysical rise of baryon density for $120 \mathrm{MeV} \leq T \leq T_{0} \mathrm{MeV}$. Moreover, the net baryon density does not vanish along with baryon chemical potential. Within months we solved the problem numerically without this approximation; the results are presented in Chap. 27 "Extreme States of Nuclear Matter", and in [1]. However, this report is the most detailed available document describing the theory behind the statistical bootstrap model of hot nuclear matter. Being distracted by the rise of the relativistic heavy ion research program, this more theoretical work was shelved to be part of a Physics Reports article, and was never formally published, not even as a TH-preprint. It is, however available (see footnote) as a CERN library archived document. The Physics Reports review that would have contained this material was never completed.

Presented by JR at the XVIIth International Winter Meeting on Nuclear Physics, Bormio, Italy, 22-27 January, 1979, pp. 531-563 in 'home' press proceedings: Editor: I. Iori, Instituto di Fizica Universita Milano, available at: http://cds.cern.ch/record/134674/files/CM-P00055555.pdf.

J. Rafelski $(\bowtie)$

Department of Physics, The University of Arizona, Tucson, AZ 85721, USA

R. Hagedorn: (deceased) CERN-TH, 1211 Geneve 23, Switzerland 


\subsection{Introduction}

Properties of nuclear matter have inspired much of the theoretical work in manybody theory during the last decades. While initially attention was focused on the saturation properties of cold nuclear matter, more recently the advent of high-energy heavy ion accelerators has stimulated work on the high temperature and density domain of the phase diagram.

There exist several main lines of approach to this complicated theoretical problem in which substantial simplifications of the actual physical circumstances are supposed. We will not review these approaches here except to say that they can be divided into two categories: (1) the nuclear matter is considered to be a non-interacting ideal gas; or (2) nuclear interactions are considered at the level of classical particle scattering.

It is immediately apparent that the interesting features of nuclear matter, such as density isomerism at high temperatures, phase transitions, condensation phenomena, etc., can hardly be discussed in the framework of the ideal gas equations of state. The fact that some kind of agreement of inclusive particle spectra in heavy ion collisions is found between theory and experiment is in fact only indicative that a thermal equilibrium is achieved in a fireball created in the collisions. To find out more about the properties of these fireballs, one has to perform more refined experiments and consider a more elaborate theory. This aim is achieved in a nonthermodynamical way in the approaches that deal with the $A_{1}+A_{2}$ many-body problem, in which each particle is followed during the collision; but it becomes virtually impossible to identify the relevant collective motion that is characteristic of phase transitions and critical phenomena.

In order to derive the physical properties of hot nuclear matter which are independent of a particular choice of the two-body and multibody interaction, we employ a technique ('bootstrap') developed for similar problems in elementary particle physics-here, however, sufficiently modified to suit the different physical environment. An additional motivation in this direction is the recent recognition that the understanding of nuclear matter at the saturation point depends very sensitively on the character of the two-body potential at short distances, which is not well defined by two-body reactions. It is possible to view the bootstrap technique only as a convenient way of introducing the relevant physical properties which cannot be so easily defined by the choice of a specific potential, but which globally might even be more important than details of the two-body force. We will concentrate on the gross features of nuclear matter that follow when we incorporate into the description the following aspects of nuclear interactions:

(i) conservation of baryon number and clustering of nucleons (i.e., attractive forces leading to many-body clusters with well-defined baryon number);

(ii) nucleon (isobar) excitations and internal cluster excitations (i.e., internal degrees of freedom that can absorb part of the energy of the system at finite temperature, thus transforming kinetic energy into mass); 
(iii) approximate extensivity of nuclear matter (volume roughly proportional to baryon number, i.e., effectively a short-range repulsion);

(iv) co-existence of a pion gas when the temperature is not equal to zero (and behaving properly even in the presence of nuclear matter);

(v) baryon-antibaryon pair creation;

(vi) 'chemical' equilibrium between all constituents of the system (nucleons, isobars, clusters, pions, etc.).

Our present work [2] should be most trustworthy in the domain of high temperatures and moderately high density, where details of the interaction, of Fermi and Bose statistics, as well as of the quark structure of nucleons, are most likely negligible. Also not considered explicitly here is the isospin of the nuclei.

An important new feature of the Statistical Bootstrap Model as we introduce it here is that the energy density in fireballs,

$$
\varepsilon_{\mathrm{FB}}:=\frac{m}{V(m)}=: A,
$$

is finite, constant, and of the order of the rest-energy density of a proton. Therefore it occurs to us that it is not reasonable to apply the thermodynamics derived from the 'bootstrap equation' beyond the point where the energy density $\varepsilon(T)$ becomes much larger than $1 / A$.

\section{Plan of the Paper}

Section 23.2. We discuss the bootstrap hypothesis first in the context of a strongly interacting pion gas. The bootstrap equation of the pion gas is solved and discussed. We write down, discuss, and solve the bootstrap equation for nuclear matter. It is much richer than that of the pion gas, which it contains as a special case.

Section 23.3. The mass spectrum and its Laplace transform are used to obtain a thermodynamic description of the system. We compute the partition functions for clustered nuclear matter.

Section 23.4. We study the properties of nuclear matter in the thermodynamic limit. Two main properties of our model are:

(a) there exists a maximum temperature, which is of the order of that of the pion gas $\left(T_{0} \approx m_{\pi}\right)$

(b) there exists at all temperatures $0 \leq T \leq T_{0}$ a critical baryon number density separating a low-density 'gas phase' from a state where a condensate and its vapor exist in equilibrium.

A numerical study is presented in which the simplest non-trivial input spectrum is assumed; the corresponding model is solved explicitly and the results are 
displayed graphically. This case shows all essential features, but it is still too far from reality to be taken as more than a qualitative prediction.

Section 23.5. Summary.

Our notation and units:

- $\hbar=c=k($ Boltzmann constant $)=1$;

- the only dimensional unit is $1 \mathrm{GeV}=1,000 \mathrm{MeV} \approx 5 \mathrm{fm}^{-1}$;

- metric: $a \cdot b \equiv a_{\mu} b^{\mu} \equiv a_{0} b_{0}-\mathbf{a} \cdot \mathbf{b}$.

Thus for example $m^{2}:=p^{2}=p_{0}^{2}-\mathbf{p}^{2}=E^{2}-\mathbf{p}^{2}$.

Remark. Throughout this paper we use only Boltzmann statistics. As the bootstrap approach leads to an extremely rich mass spectrum, it is almost irrelevant whether a particular cluster or particle is a boson or a fermion or a Boltzmannion: it (almost) never happens that two equal clusters occupy the same state.

\subsection{The Statistical Bootstrap Method in Particle and Nuclear Physics}

\section{The Statistical Bootstrap Model in Particle Physics}

The motivation for a statistic bootstrap model in particle physics comes from two sources:

(a) the abundant production of particles in high-energy $p p$ collisions, and a momentum distribution of these particles which suggests that there might be some analogy to black-body radiation emitted from moving sources;

(b) the apparent existence of intermediate states in which lumps of highly excited hadronic matter ('fireballs') are staying together before decaying.

Thus it was tempting to describe the particle production process as pion black-body radiation emitted from one or several fireballs with a volume $v_{0} \approx 4 \pi / 3 m_{\pi}^{3}$. This idea was first proposed by Koppe [3] and it is called the Fermi statistical model [4]. As for a statistical-thermodynamical description, the density of states $\sigma(E)$ is necessary and sufficient; we may express the Koppe-Fermi approach as follows:

$$
\frac{v_{0} \sigma_{0}(E)}{(2 \pi)^{3}}=\sum_{n=1}^{\infty} \frac{1}{n !} \int \delta\left(E-\sum_{i=1}^{n} \sqrt{\mathbf{p}_{i}^{2}+m_{\pi}^{2}}\right) \delta^{3}\left(\sum_{i=1}^{n} \mathbf{p}_{i}\right) \prod_{i=1}^{n} \frac{v_{0} \mathrm{~d}^{3} p_{i}}{(2 \pi)^{3}} .
$$

This is nothing else than the phase-space density of a pion gas with free particle creation. If we put $m=0$ and multiply by 2 for the two helicity states of a light quantum, we obtain from Eq. (23.2) all the usual formulas of the electromagnetic black-body radiation (Planck's law) in the Boltzmann limit. 
The next important idea was to admit particles other than just pions, and in particular resonant states of pions, just as if they were stable particles [5]. Not knowing which ones should be admitted and how many there are, we might put them in a mass spectrum of admissible input particles $\rho_{\text {in }}(m)$. The pion contributes to $\rho_{\text {in }}(m)$ a $\delta$-function $\delta\left(m-m_{\pi}\right)$; resonances contribute smeared-out $\delta$-functions. For the moment, $\rho_{\text {in }}(m)$ is a function which represents our (incomplete) knowledge of the true mass spectrum $\rho(m)$.

Introducing also relativistically invariant notation with a four-volume $V^{\mu}=$ $v_{0} u^{\mu}, u^{2}=1$, and $\delta_{0}\left(p^{2}-m^{2}\right)=\delta\left(p^{2}-m^{2}\right) \theta\left(p_{0}\right)$,

$$
\begin{aligned}
\sigma\left(p^{2}, p \cdot V\right)= & \frac{2 p \cdot V}{(2 \pi)^{3}} \delta_{0}\left(p^{2}-m_{\pi}^{2}\right) \\
& +\sum_{n=2}^{\infty} \frac{1}{n !} \int \delta^{4}\left(p-\sum_{i=1}^{n} p_{i}\right) \prod_{i=1}^{n} \frac{2 p_{i} \cdot V}{(2 \pi)^{3}} \rho_{\mathrm{in}}\left(m_{i}\right) \delta_{0}\left(p_{i}^{2}-m_{i}^{2}\right) \mathrm{d}^{4} p_{i} \mathrm{~d} m_{i} .
\end{aligned}
$$

This is a relativistically invariant equation for the density of states $\sigma\left(p^{2}, p \cdot V\right)$ in $\left\{p, \mathrm{~d}^{4} p\right\}$ of a gas in which the interaction manifests itself via creation and absorption of Boltzmann pions and their excited states contained in $\rho_{\text {in }}(m)$.

Note that we have restricted the one-particle state to have the pion mass. Higher mass 'one-particle states' are already contained in the sum, namely when in any of its terms all $p_{i} \rightarrow m_{i}$. Our new equation for $\sigma\left(p^{2}, p \cdot V\right)$ describes the density of states of a many-component gas: each species of particle contained in $\rho_{\text {in }}(m)$ is present in the gas. All these components are in 'chemical' equilibrium; neither the total particle number nor that of any of the various components is fixed.

The key idea that leads to the hadronic bootstrap is the observation that the quantity $\sigma\left(p^{2}, p \cdot V\right)$ can be related to the mass spectrum $\rho(m)$. Suppose we could insert the true mass spectrum $\rho(m)$ into Eq. (23.3). Then $\sigma\left(p^{2}, p \cdot V\right)$ would be the density of states of a 'fireball' of hadronic dimension built up from all strongly interacting particles in statistical equilibrium. Such a fireball is itself a highly excited hadron with mass $m=\sqrt{p^{2}}$. For reasons of consistency, it should then be admitted as a constituent particle in fireballs of larger mass. Hence it should already be present in the true $\rho(m)$. As both $\sigma\left(p^{2}, p \cdot V\right)$ and $\rho(m)$ are densities of states, it follows that if $\rho(m)$ is the true mass spectrum, $\sigma\left(p^{2}, p \cdot V\right)$ is itself (apart from some minor kinematical differences) the true mass spectrum at $m=\sqrt{p^{2}}$. This statement establishes a new relation between $\rho$ and $\sigma$, leading to an integral equation, the bootstrap equation. Physically, it is equivalent to the postulate that resonances and fireballs are one and the same and that fireballs consist of fireballs.

In order to find the precise relation between $\rho(m)$ and $\sigma\left(p^{2}, p \cdot V\right)$, we consider the conceptual differences between them as exhibited by Eq. (23.3): while $\rho(m)$ counts all hadrons (as given, for example, in the tables of the Particle Data Group [6]) as being at rest in their own confining volume, $\sigma\left(p^{2}, p \cdot V\right)$ is the density of states of an object with mass $m=\sqrt{p^{2}}$ allowed to move freely in its confining volume instead of being at rest. This fact is also reflected by the dependence of $\sigma$ on the scalar 
product $p \cdot V$. Thus $\sigma$ counts more states than $\rho$ (and contains more information). In order to relate $\sigma$ to $\rho$, we restrict this freedom by requiring that $p^{\mu}$ and $V^{\mu}$ be parallel four-vectors, i.e., have a common rest frame. Then $\sigma\left(p^{2}, p \cdot V\right) \rightarrow \bar{\sigma}\left(p^{2}=m^{2}, v_{0}\right)$ and the left-hand side represents the internal density of states of a system of mass $m$ at rest in its own volume $v_{0}$; this density begins with $\left[v_{0} /(2 \pi)^{3}\right] \delta\left(m-m_{\pi}\right)$ and has a continuum for $m>2 m_{\pi}$. It therefore might be considered as proportional to an averaged mass spectrum (the true one is not yet continuous at $m \geq 2 m_{\pi}$ ), which asymptotically becomes physically equivalent to $\rho(m)$. We thus have

$$
\left.\sigma\left(p^{2}, p \cdot V\right)\right|_{p \| V}=\bar{\sigma}\left(m^{2}, v_{0}\right):=\frac{v_{0}}{(2 \pi)^{3}} \rho_{\mathrm{av}}(m) \underset{m \rightarrow \infty}{\longrightarrow} \frac{v_{0}}{(2 \pi)^{3}} \rho(m) .
$$

The precise relationship between $\rho_{\mathrm{av}}, \rho_{\mathrm{in}}$, and $\rho$ will not concern us here-indeed, taking $\rho_{\mathrm{av}}=\rho_{\mathrm{in}}=\rho$, we will find, solving Eq. (23.3), the result

$$
\rho(m) \underset{m \rightarrow \infty}{\sim} \frac{c}{m^{a}} \mathrm{e}^{m / T_{0}}, \quad T_{0} \approx m_{\pi}, \quad \frac{3}{2}<a<\frac{7}{2},
$$

where $T_{0}$ is a 'limiting temperature' and where the values of $a$ and $T_{0}$ depend on the version of Eq. (23.3) chosen. We will now show how to solve bootstrap equations (23.3) and (23.4) and prove Eq. (23.5).

\section{Solution of the Bootstrap Equation}

For illustrative purposes, let us here consider the bootstrap equation in its simplest form, as proposed by Yellin [7]:

$$
B \tau\left(p^{2}\right)=B \delta_{0}\left(p^{2}-m_{\pi}^{2}\right)+\sum_{n=2}^{\infty} \frac{1}{n !} \int \delta^{4}\left(p-\sum_{i=1}^{n} p_{i}\right) \prod_{i=1}^{n} B \tau\left(p_{i}^{2}\right) \mathrm{d}^{4} p_{i},
$$

where the relation between $\tau$ and $\rho$ is

$$
\tau\left(m^{2}\right) \mathrm{d} m^{2}=\rho(m) \mathrm{d} m,
$$

and $B$ is a parameter of the model, related to the volume $v_{0}$ by a dimensional relation $B \sim v_{0} m_{\pi}$.

The standard method of solving Eq. (23.6) is by Laplace transformation. We introduce two Lorentz-invariant functions:

$$
\begin{aligned}
& \Phi(\beta):=\int B \tau\left(p^{2}\right) \mathrm{e}^{-\beta_{\mu} p^{\mu}} \mathrm{d}^{4} p \\
& \varphi(\beta):=\int B \delta_{0}\left(p^{2}-m_{\pi}^{2}\right) \mathrm{e}^{-\beta_{\mu} p^{\mu}} \mathrm{d}^{4} p=2 \pi B m_{\pi}^{2} \frac{K_{1}\left(\beta m_{\pi}\right)}{\beta m_{\pi}},
\end{aligned}
$$

where $\beta^{0}>0, \beta=\left(\beta_{\mu} \beta^{\mu}\right)^{1 / 2}$. 
Taking the Laplace transform, as defined by Eq. (23.8), of Eq. (23.6), we obtain

$$
\Phi(\beta)=\varphi(\beta)+\mathrm{e}^{\Phi(\beta)}-\Phi(\beta)-1 .
$$

Equation (23.9) can be written

$$
\varphi=2 G-\mathrm{e}^{G}+1
$$

and the problem is to invert this equation; that is, to find $G(\varphi)=\Phi(\beta)$. The easiest way to do this is a graphical solution by first plotting $\varphi(G)$ and then considering the curve as $G(\varphi)$. By expanding $\exp (G)$, we see that $\varphi(G)=G+\cdots$; with growing $G$, the exponential function takes the lead and $\varphi(G)$ goes exponentially to $-\infty$. The maximum lies at $G_{0}=\ln 2$ and has the value $\varphi_{0}=\ln 4-1 ; \varphi^{\prime \prime}\left(G_{0}\right) \neq 0$ (see Fig. 17.5a). The graphical solution is presented in Fig. 17.5b. From the figure and $\varphi^{\prime \prime}\left(G_{0}\right) \neq 0$, it follows that $G(\varphi)$ has a square root branch point at $\varphi_{0}$ and is complex for $\varphi>\varphi_{0}$ [8]. We note that $\varphi_{0}=\ln 4-1$ corresponds to the value $\beta_{0} \approx 1 / m_{\pi}$ in Eq. (23.8b); we also note that $\varphi$ increases monotonically with $1 / \beta^{1}$.

Thus in Fig. 17.5b, the interval $\varphi \in\left\{0, \varphi_{0}\right\}$ corresponds uniquely to $\beta \in\left\{\infty, \beta_{0}\right\}$. Given $\varphi$, we could invert Eq. (23.8a); however, we can obtain the physically interesting information about $\tau$ without an explicit inversion.

Since $\int \delta_{0}\left(p^{2}-m^{2}\right) \mathrm{d} m^{2}=1$, we may write

$$
\begin{aligned}
\Phi(\beta) & =\int \tau\left(m^{2}\right) \mathrm{d} m^{2} \int B \delta_{0}\left(p^{2}-m^{2}\right) \mathrm{e}^{-\beta_{\mu} p^{\mu}} \mathrm{d}^{4} p \\
& =\frac{2 \pi B}{\beta} \int \tau\left(m^{2}\right) m K_{1}(\beta m) \mathrm{d} m^{2} .
\end{aligned}
$$

As we have just seen, $G(\varphi)$ has a square root branch point at $\varphi_{0}$, and so has $\Phi(\beta)$ at $\beta_{0}$, since $\varphi$ is monotonic in $\beta$. Since $K_{1}(m \beta)$ behaves like $\exp (-\beta m)$ for $m \rightarrow \infty$, Eq. (23.11) can yield a singularity of $\Phi(\beta)$ only if $\tau\left(\mathrm{m}^{2}\right)$ grows asymptotically like $\exp \left(\beta_{0} m\right)$; a square root branch point requires

$$
\tau\left(m^{2}\right) \sim \frac{c}{m^{3}} \mathrm{e}^{\beta_{0} m},
$$

which illustrates the relation (23.5) for $a=6 / 2$.

From Eq. (23.10), a Taylor expansion for $G$ around $\varphi=0$ can be found, with the convergence radius $\varphi_{0}$ :

$$
G(\varphi)=\sum_{n=1}^{\infty} g_{n} \varphi^{n}
$$

\footnotetext{
${ }^{1}$ The surprisingly complex analytical structure of this seemingly simple bootstrap function is further explored in: R. Hagedorn and J. Rafelski, "Analytic Structure and Explicit Solution of an Important Implicit Equation,” Commun. Math. Phys. 83, 563 (1982).
} 
which leads to an analytic form of $\tau\left(p^{2}\right)$ :

$$
B \tau\left(p^{2}\right)=\sum_{n=1}^{\infty} g_{n} \Omega_{n}\left(p^{2}, B\right),
$$

where $\Omega_{n}$ is the $n$-pion invariant momentum space (IMS) integral

$$
\Omega_{n}\left(p^{2}, B\right):=\int \delta^{4}\left(p-\sum_{i=1}^{n} p_{i}\right) \prod_{i=1}^{n} B \delta_{0}\left(p_{i}^{2}-m_{\pi}^{2}\right) \mathrm{d}^{4} p_{i} .
$$

The IMS integrals are well-known functions for which powerful computer programs exist. Therefore, Eq. (23.15) is very useful at not too large $p^{2}$, since the sum has actually only a finite number of terms-it is cut off at $n \leq \sqrt{p^{2}} / m_{\pi}$ by the momentum $\delta^{4}$-function and by the condition $p_{0} \geq m_{\pi}$.

Had we used the IMS measure in Eq. (23.3), the density of states of the pion gas would have read

$$
\sigma_{\mathrm{IMS}}\left(p^{2}\right)=\sum_{n=1}^{\infty} \frac{1}{n !} \Omega_{n}\left(p^{2}, B\right)
$$

while now we have Eq. (23.14).

It can be seen that the rapidly decreasing $1 / n$ ! has been replaced by the (exponentially increasing!) $g_{n}$. Thus the $\Omega_{n}$ in Eq. (23.16) have been multiplied by $n ! g_{n}$, which is the total number of possible ways to cluster $n$ objects recursively (admitting clusters of clusters).

It remains to determine the coefficients $g_{n}$. This is done most simply by considering the first-order differential equation that $G$ satisfies:

$$
1=\frac{\mathrm{d} G}{\mathrm{~d} \varphi}(\varphi+1-2 G) .
$$

Inserting Eq. (23.13), we find the recursion relation

$$
g_{n}=-\frac{n-1}{n} g_{n-1}+\sum_{k=1}^{n-1} g_{k} g_{n-k}, \quad g_{0}=0, \quad g_{1}=1 .
$$

Given Eqs. (23.13), (23.14), and (23.18), the bootstrap equation (23.6) can be considered as solved. 


\section{The Nuclear Matter Bootstrap Equation}

According to the aims described in Sect.23.1, we now generalize the bootstrap equation (23.6) to the case of nuclear matter. We postulate the following bootstrap equation for the level density of 'nuclear clusters' with baryon number $b \in$ $(-\infty, \infty)$ :

$$
\begin{aligned}
& \frac{2 p \cdot V}{(2 \pi)^{3}} \sigma(p, v, b)= \delta^{4}\left(V-V_{b}\right) C_{b} \frac{2 p \cdot V_{b}}{(2 \pi)^{3}} \delta_{0}\left(p^{2}-M_{b}^{2}\right) \\
&+\sum_{k=2}^{\infty} \frac{1}{k !} \int \sum_{\left\{b_{i}\right\}} \delta_{k}\left(b-\sum_{i=1}^{k} b_{i}\right)\left(p-\sum_{i=1}^{k} p_{i}\right)\left(V-\sum_{i=1}^{k} V_{i}\right) \\
& \times \prod_{i=1}^{k} \frac{2 p_{i} \cdot V_{i}}{(2 \pi)^{3}} \sigma\left(p_{i}, V_{i}, b_{i}\right) \mathrm{d}^{4} p_{i} \mathrm{~d}^{4} V_{i}
\end{aligned}
$$

Equation (23.19) is not a single bootstrap equation, but a member (with baryon number $b$ ) of an infinite set of coupled integral equations, each having its own input term. The $(k !)^{-1}$ is necessary for correct counting. The non-vanishing pion and nucleon mass ensure that, for any finite $p^{2}$, the set (23.19) has only a finite number of equations: $\left|b_{\max }\right| \leq \sqrt{p^{2}} / m_{p}$. Therefore the solutions for any finite $p^{2}$ can (in principle) be built up iteratively by starting with $4 m_{\pi}^{2} \leq p^{2} \leq m_{p}^{2}$ and by increasing this interval stepwise to include higher and higher $|b|$. This, incidentally, also allows us to prove that, for any $p, V, b$, Eq. (23.19) have a physical solution.

This equation fulfills the requirements set up in Sect. 23.1:

1. Conservation of baryon number and clustering of nucleons. The baryon number (number of baryons minus number of antibaryons) is conserved with the help of the Kronecker $\delta_{K}\left(b-\sum b_{i}\right)$ function. The infinite set of density functions $\sigma(p, V, b)$ corresponds to the admission of nucleon clusters with any baryon number $b$, four-momentum $p$, and four-volume $V$.

2. Nucleon (isobar) excitation and internal cluster excitation. Internal cluster excitation is contained in the $p^{2}=m^{2}$ dependence of $\sigma(p, V, b)$, and singlenucleon (isobar) excitation is contained in the same way in $\sigma(p, V, b=1)$.

3. Extensivity of nuclear matter. This is ensured by the volume $\delta^{4}$-function.

4. Co-existence of a pion gas. This is contained in the equation with $b=0$, and in all others by the presence of factors $\sigma\left(p_{i}, V_{i}, b_{i}=0\right)$ on the right-hand side.

5. Baryon-antibaryon pair creation (and annihilation). This is built in by allowing $-\infty<b_{i}, b<\infty$. Then on the right-hand side an arbitrary number of clusters $\left(\sum b_{i}\right)$ and anticlusters $\left(-\sum \bar{b}_{i}\right)$ may occur. 
6. 'Chemical equilibrium' between all constituents. This is expressed by the infinite set of coupled integral equations (23.19), which allows all multibody reactions between clusters $Q_{i}$,

$$
Q_{1}+Q_{2}+\cdots+Q_{n} \rightleftarrows Q_{1}^{\prime}+Q_{2}^{\prime}+\cdots+Q_{n}^{\prime}
$$

compatible with $b$ and $p$ conservation.

The input terms, except that for $b=0$ (pion) and for $b=1$ (nucleon), specify particular features of the model, namely:

(a) Details of nuclear interaction may be represented by giving clusters (e.g., alpha particles) a special weight.

(b) Equation (23.19) deal with Boltzmann particles without charge and spin. Introducing spin, isospin, and statistics would be possible but complicated. We can obtain a similar physical effect by assigning to an input nucleus of baryon number $b$ and volume $V_{b}$ a mass $M_{b}$ which is different from $b \cdot m_{p}$.

\section{The Mass Spectrum for Nuclear Matter}

We introduce

$$
\frac{2 p \cdot V}{(2 \pi)^{3}} \sigma(p, V, b)=\delta^{4}\left(V-V(m, b) \frac{p}{m}\right) \tilde{B}\left(p^{2}, b\right) \tilde{\tau}\left(p^{2}, b\right),
$$

where the function $\tilde{B}$ describes the $p^{2}, b$ dependence of the $V \cdot p$ term, while $\tilde{\tau}$ describes that of $\sigma$. We now rewrite Eq. (23.19), integrate over the $\prod \mathrm{d}^{4} V$, and require that all volume $\delta^{4}$-functions have the same argument. We find the condition

$$
\sum_{i=1}^{n}\left[\frac{V\left(m_{i}, b_{i}\right)}{m_{i}}-\frac{V(m)}{m}\right]_{\mu} p_{i}^{\mu}=0
$$

from which it follows that, for all $i$,

$$
V\left(m_{i}, b_{i}\right)=\mathscr{A} m_{i}
$$

The constant $\mathscr{A}$ is independent of $i$ and is therefore a parameter of the theory; $\mathscr{A}^{-1}$ is the constant energy density in the natural volume $V$ : thus it is about $1 / 7 m_{\mathrm{N}} / \mathrm{fm}^{3}=$ $130 \mathrm{MeV} / \mathrm{fm}^{3}$. We further find that

$$
\tilde{B}\left(m^{2}, b\right):=\frac{2 V(m, b)}{(2 \pi)^{3}} m=\frac{2 \mathscr{A} m^{2}}{(2 \pi)^{3}}=\tilde{B}\left(m^{2}\right)
$$

is independent of $b$. 
The volume $\delta^{4}$-function can now be factored out on both sides of Eq. (23.19), and what remains is a new bootstrap equation for the function $\tilde{B} \cdot \tilde{\tau}\left(p^{2}, b\right)$ :

$$
\begin{aligned}
\tilde{B}\left(p^{2}\right) \tilde{\tau}\left(p^{2}, b\right)= & C_{b} \tilde{B}\left(M_{b}^{2}\right) \delta_{0}\left(p^{2}-M_{b}^{2}\right) \\
& +\sum_{k=2}^{\infty} \frac{1}{k !} \int \sum_{\left\{b_{i}\right\}} \delta_{k}\left(b-\sum_{i=1}^{k} b_{i}\right) \delta^{4}\left(p-\sum_{i=1}^{k} p_{i}\right) \prod_{i=1}^{k} \tilde{B}\left(p_{i}^{2}\right) \tilde{\tau}\left(p_{i}^{2}, b_{i}\right) \mathrm{d}^{4} p_{i} .
\end{aligned}
$$

The essential step now consists in the proper extraction of the mass spectrum $\tau\left(p^{2}, b\right)$ from the function $\tilde{\tau}$. Motivated by the form (23.6) of the bootstrap equation, we chose here

$$
\tau\left(p^{2}, b\right):=\frac{\tilde{B}\left(p^{2}\right)}{\tilde{B}\left(M_{b}^{2}\right)} \tilde{\tau}\left(p^{2}, b\right),
$$

since we can write the bootstrap equation for the mass spectrum as

$$
\begin{aligned}
B_{b} \tau\left(p^{2}, b\right)= & C_{b} B_{b} \delta_{0}\left(p^{2}-M_{b}^{2}\right) \\
& +\sum_{k=2}^{\infty} \frac{1}{k !} \int \sum_{\left\{b_{i}\right\}} \delta_{k}\left(b-\sum_{i=1}^{k} b_{i}\right) \delta^{4}\left(p-\sum_{i=1}^{k} p_{i}\right) \prod_{i=1}^{k} B_{b} \tau\left(p_{i}^{2}, b_{i}\right) \mathrm{d}^{4} p_{i},
\end{aligned}
$$

where

$$
B_{b}:=\tilde{B}\left(M_{b}^{2}\right)=\frac{2 \mathscr{A} M_{b}^{2}}{(2 \pi)^{3}} .
$$

We would like to emphasize that the choice (23.25) leading to Eq. (23.26) is arbitrary. Another very likely choice is to take $\tilde{\tau}$ as the physical mass spectrum. As we have found recently, this significantly simplifies our final formula but complicates the numerical evaluation. Throughout this paper, we will constrain our work to the mass spectrum defined through Eq. (23.25).

The bootstrap equation (23.26) is much richer than that for the pion gas; we have allowed the presence of arbitrarily complicated clusters characterized by the baryonic number $b_{i}$. For $b=0$, we have a description of meson fireballs; but in order to understand these fireballs properly, especially when baryon-antibaryon clusters are among their constituents, we have to obtain a solution for the function $\tau$ for all values of $b$. 


\section{Laplace and L-Transforms of the Mass Spectrum}

In order to solve the nuclear bootstrap equation, a treatment of the $b$ dependence is necessary. This is done by defining the 'L-transform' ${ }^{\text {: }}$

$$
L[f(b)]:=\sum_{b=-\infty}^{\infty} \lambda^{b} f(b):=f_{\lambda}(\lambda)
$$

Hence $f_{\lambda}(\lambda)=L[f(b)]$ is the generating function of $f(b)$. We multiply the entire bootstrap equation by $\lambda^{b}$ and sum over $b$. Defining the L-transform of $\tau\left(p^{2}, b\right)$ and of the input term, respectively,

$$
\begin{aligned}
B_{\mathrm{N}} \tau_{\lambda}\left(p^{2}, \lambda\right) & :=\sum_{b=-\infty}^{\infty} \lambda^{b} B_{b} \tau\left(p^{2}, b\right), \\
B_{\mathrm{N}} \tau_{0 \lambda}\left(p^{2}, \lambda\right) & :=B_{\pi} \delta_{0}\left(p^{2}-m_{\pi}^{2}\right)+\sum_{b=1}^{\infty} C_{b} B_{b}\left(\lambda^{b}+\lambda^{-b}\right) \delta_{0}\left(p^{2}-M_{b}^{2}\right),
\end{aligned}
$$

where

$$
B_{1}=B_{\mathrm{N}}, \quad M_{1}=m_{\mathrm{N}}
$$

we find that the bootstrap equation takes the form of the pion bootstrap equation (23.6), but with a much more involved input function $\tau_{0 \lambda}$,

$$
\tau_{\lambda}\left(p^{2}, \lambda\right)=\tau_{0 \lambda}\left(p^{2}, \lambda\right)+\sum_{k=2}^{\infty} \frac{1}{k !} \int \delta^{4}\left(p-\sum_{i=1}^{k} p_{i}\right) \prod_{i=1}^{k} \tau_{\lambda}\left(p_{i}^{2}, \lambda\right) \mathrm{d}^{4} p_{i} .
$$

That illustrates the general bootstrap philosophy that the input function characterizes the 'raw material', while the integral equation imposes the dynamics on it. The dynamics should be more or less independent of what the raw material is (but it will depend on kinematics, statistics, etc.).

In order to solve Eq. (23.29), we introduce the Laplace transforms of $\tau_{\lambda}$ and $\tau_{0 \lambda}$ :

$$
\Phi(\beta, \lambda):=\int B_{\mathrm{N}} \tau_{\lambda}\left(p^{2}, \lambda\right) \mathrm{e}^{-\beta \cdot p} \mathrm{~d}^{4} p=: \sum_{b=-\infty}^{\infty} \lambda^{b} \Phi_{b}(\beta, b),
$$

\footnotetext{
${ }^{2}$ We use the expression 'L-transform' to stress the formal analogy with the Laplace transform: $\mathrm{L}$ is the discrete counterpart of $\mathscr{L}$.
} 


$$
\varphi(\beta, \lambda):=\int B_{\mathrm{N}} \tau_{0 \lambda}\left(p^{2}, \lambda\right) \mathrm{e}^{-\beta \cdot p} \mathrm{~d}^{4} p
$$

In analogy with the case of pionic bootstrap, we now find the bootstrap equation [see Eq. (23.9)] considering the Laplace transform of Eq. (23.29):

$$
\Phi(\beta, \lambda)=\varphi(\beta, \lambda)+\exp [\Phi(\beta, \lambda)]-\Phi(\beta, \lambda)-1
$$

For the input function $\varphi$, we have explicitly

$$
\begin{gathered}
\varphi(\beta, \lambda)=\sum_{b=-\infty}^{\infty} \lambda^{b} \varphi_{b}(\beta, b), \\
\varphi_{b}(\beta, b)=\varphi_{b}(\beta,-b)=C_{b} B_{b} 2 \pi M_{b}^{2} \frac{K_{1}\left(\beta M_{b}\right)}{\beta M_{b}} .
\end{gathered}
$$

In Fig. 23.1, we give a short summary of the relations between the functions arising from $\tau$ through application of $\mathscr{L}$ and L-transforms.

The bootstrap equation (23.32) for the doubly transformed function $\Phi(\beta, \lambda)$ has a real solution wherever in the $(\beta, \lambda)$ plane the input function $\varphi<\varphi_{0}=\ln 4-1$ (see Fig. 17.5a, b). Thus along a curve $\beta_{\mathrm{c}}=f\left(\lambda_{\mathrm{c}}\right)$ in the $(\beta, \lambda)$ plane defined as the boundary of this domain $\left(\beta_{\mathrm{c}}, \lambda_{\mathrm{c}}\right)=\varphi_{0}$, a qualitative change in the behavior of the properties of nuclear matter may occur. Quite aside from the physical questions, we have to ask for a mathematical solution of the bootstrap equation beyond this boundary line. As we have previously argued by a recursive argument, a physical solution for $\tau\left(p^{2}, b\right)$ exists for any $p^{2}$. Our $\Phi(\beta, \lambda)$ is the Laplace-L-transform of $B_{b} \tau\left(p^{2}, b\right)$, which does not exist in this form everywhere in $(0 \leq \beta<\infty) \otimes(1 \leq$ $\lambda<\infty)$. However, once defined in a domain where it does exist, it fulfills Eq. (23.4), which then permits analytical continuation of $\Phi(\beta, \lambda)$ beyond the limit $\varphi=\varphi_{0}$ in

Fig. 23.1 Relations between the mass spectrum $\tau\left(p^{2}, b\right)$ and its Laplace $(\mathscr{L})$ and L-transforms

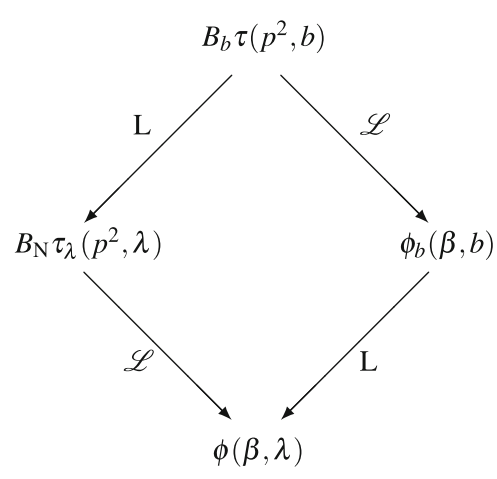


the whole (complex $\beta) \otimes($ complex $\lambda$ ) domain. Thus using the methods of complex analysis, we will be in a position to study the new phases in the future.

We remark here that the analytical continuation beyond $\varphi_{0}$ has never been considered in the case of pionic bootstrap, since there this limit on $\varphi$ led to a limiting temperature; the energy of fireballs diverged at this point and made a transition from our world to the new domain impossible. Now the presence of baryons changes this - the introduction of $\lambda$ leads to the existence of a new region with $T<T_{0}$ but $\varphi>\ln 4-1$. We will find in our present model again a boundary $T=T_{0}$, at which the energy density diverges-but this limit is not at $\varphi=\ln 4-1$, except when $\lambda=1$.

\subsection{Thermodynamics}

In Sect. 23.2, we solved the bootstrap equation with the help of the Laplace transformation. The same mathematical procedure is used in statistical thermodynamics to obtain the partition function from the density of states. This coincidence has the effect that the Laplace transform $\Phi(\beta)$ of the mass spectrum $\tau\left(p^{2}\right)$ and the Laplace transform $Z(\beta, V)$ of the density of states of a thermodynamical system containing particles with the mass spectrum $\tau\left(\mathrm{m}^{2}\right)$ can easily be confounded. We expect a relation between $\Phi(\beta)$ and $Z(\beta, V)$ - and we will exploit it below-but conceptually these two quantities are different.

\section{The Partition Functions of the One-Component Ideal Gas}

Consider an ideal relativistic Boltzmann gas with one sort of particle of mass $m$ enclosed in an arbitrary, macroscopic external volume $V_{\mu}^{\mathrm{ex}}$. The number of states $\left\{p, \mathrm{~d}^{4} p\right\}$ of one particle in the four-volume $V_{\mu}^{\mathrm{ex}}$ is

$$
\frac{2 V_{\mu}^{\mathrm{ex}} p^{\mu}}{(2 \pi)^{3}} \delta_{0}\left(p^{2}-m^{2}\right) \mathrm{d}^{4} p
$$

Thus the one-particle partition function $Z_{1}^{(0)}$ (in which the superscript denotes 'noninteracting') is defined by

$$
Z_{1}^{(0)}\left(\beta, V^{\mathrm{ex}}\right):=\int \frac{2 V_{\mu}^{\mathrm{ex}} p^{\mu}}{(2 \pi)^{3}} \delta_{0}\left(p^{2}-m^{2}\right) \mathrm{e}^{-\beta_{\mu} p^{\mu}} \mathrm{d}^{4} p .
$$


Here the four-volume $V_{\mu}^{\text {ex }}$ is an arbitrary external parameter ${ }^{3}$ [a box of arbitrary volume $V^{\mathrm{ex}}=\left(V_{\mu} V^{\mu}\right)^{1 / 2}$ having an arbitrary four-velocity], while before, in Sect. 23.2, we took the volume to be the dynamically determined proper comoving volume of the particle. $\beta_{\mu}$ has now the meaning of the inverse temperature fourvector, where (the Lorentz invariant) $T=(\beta \cdot \beta)^{-1 / 2}$ is the temperature in the rest frame of the thermometer. $Z_{1}^{(0)}$ is by construction a function of the invariants $\beta^{2}$, $V_{\mathrm{ex}}^{2}$, and $\beta^{\mu} V_{\mu}^{\mathrm{ex}}$. As it seems not very useful to consider a description where the thermometer moves (fast) with respect to the container of a gas, we take here $\beta^{\mu}$ parallel to $V_{\mathrm{ex}}^{\mu}$. We then obtain in the common rest frame of $\beta^{\mu}$ and $V^{\mu}$,

$$
Z_{1}^{(0)}(\beta, V)=\frac{V m^{3}}{2 \pi^{2}} \frac{K_{2}(\beta m)}{\beta m} .
$$

[Notice the difference with Eq. (23.8b).]

From the one-particle partition function, the $N$-Boltzmann-particle partition is found:

$$
Z_{N}^{(0)}(\beta, V)=\frac{1}{N !} Z_{1}^{(0)}(\beta, V)^{N}
$$

The grand canonical partition function is then

$$
Z^{(0)}(\beta, V, \lambda):=\sum_{n=0}^{\infty} \lambda^{n} Z_{n}^{(0)}(\beta, V)^{n}=\mathrm{e}^{\lambda Z_{1}^{(0)}(\beta, V)}
$$

with $\lambda$ being the fugacity. From $Z^{(0)}(\beta, V, \lambda)$ nearly all relevant quantities can be found by logarithmic differentiation, in particular

$$
\begin{aligned}
\varepsilon^{(0)}(\beta, V, \lambda) & :=-\frac{1}{V} \frac{\partial}{\partial \beta} \ln Z^{(0)}(\beta, V, \lambda)=\text { energy density } \\
P^{(0)}(\beta, V, \lambda) & :=\frac{T}{V} \ln Z^{(0)}(\beta, V, \lambda)=\text { pressure } \\
n^{(0)}(\beta, V, \lambda) & :=\frac{\lambda}{V} \frac{\partial}{\partial \lambda} \ln Z^{(0)}(\beta, V, \lambda)=\text { particle number density }
\end{aligned}
$$

and so on. We introduce the relativistic chemical potential (equal to $\mu_{\text {non-rel }}+m$ ) by $\lambda=\mathrm{e}^{\beta \mu} ; \mu=0(\lambda=1)$ corresponds to black-body radiation of quanta with rest mass $m$.

\footnotetext{
${ }^{3}$ We will often drop the superscript 'ex' on $V$ when the meaning is unambiguous.
} 


\section{The Strongly Interacting Pion Gas}

The basic hypothesis is that in many instances an assembly of strongly interacting particles (of one kind ${ }^{4}$ ) enclosed in an arbitrary volume at arbitrary temperature and chemical potential may be described

- either as a multitude of particles of one kind with a complicated interaction,

- or as a non-interacting phase consisting of an infinity of different species with a mass spectrum appropriate to the interaction in question.

This implies that, if the mass spectrum of the interaction is known, replacing the interacting particles by an ideal infinite-component phase and weighting the different components according to the mass spectrum generates the same distortion of phase space as the interaction would do. An example is, for instance, a dilute He gas. Usually, this is not described as an assembly of protons, neutrons, and electrons with a Hamiltonian containing QED and strong interactions; instead, one uses the mass spectrum (here essentially one state with mass, spin, etc., of ${ }^{4} \mathrm{He}$ ) and calculates the properties of an ideal Bose gas of He atoms, considering the latter as elementary.

Taking now the attitude that the statistical bootstrap model has provided us with the correct spectrum, the corresponding statistical thermodynamics of strongly interacting particles follows from the formulas of the ideal gas given in Sect. 23.3, now generalized to include the mass spectrum. The one-particle phase-space measure (23.35) now becomes the 'one-fireball' phase-space measure:

$$
\mathrm{d}^{4} p \sigma_{1}(p, V)=\frac{2 V_{\mu}^{\mathrm{ex}} p^{\mu}}{(2 \pi)^{3}} \mathrm{~d}^{4} p \int \mathrm{d} m^{2} \tau\left(m^{2}\right) \delta_{0}\left(p^{2}-m^{2}\right)=\frac{2 V_{\mu}^{\mathrm{ex}} p^{\mu}}{(2 \pi)^{3}} \tau\left(p^{2}\right) \mathrm{d}^{4} p .
$$

Accordingly, we find the 'one-fireball' partition function

$$
Z_{1}(\beta, V)=\int \frac{2 V_{\mu}^{\mathrm{ex}} p^{\mu}}{(2 \pi)^{3}} \tau\left(p^{2}\right) \mathrm{e}^{-\beta_{\mu} p^{\mu}} \mathrm{d}^{4} p
$$

Recalling Eq. (23.8a), we find in the common rest frame of $V_{\mu}^{\mathrm{ex}}$ and $B_{\mu}$,

$$
Z_{1}(\beta, V)=-\frac{2 V^{\mathrm{ex}}}{B(2 \pi)^{3}} \frac{\partial \Phi(\beta)}{\partial \beta}
$$

We can now proceed in the same manner as in Eqs. (23.37)-(23.39), which follow now for the interacting particles, dropping the upper index (0). However, in Eq. (23.39), $n$ is now the average number of fireballs present. For this $n(\beta, V, \lambda)$,

\footnotetext{
${ }^{4}$ The generalization to several different species is straightforward.
} 
we have the ideal gas equation (due to the linearity of $\ln Z$ in $\lambda$ ):

$$
P=n T,
$$

while the corresponding equation in terms of the average number of pions (contained in all these fireballs together) would look horribly complicated. This result (23.43), which in the framework of this model is exact, shows once more how simple things become once the interaction is hidden in the mass spectrum.

\section{Physics Near $T_{0}$}

We have seen how the bootstrap equation provides us with the function $\Phi(\beta)$, from which $Z_{1}(\beta, V, \lambda)=\ln Z(\beta, V, \lambda)$ can be calculated; $Z_{1}(\beta, V, \lambda)$ then serves as the generating function for physical quantities. In all versions of the statistical bootstrap model, we find an exponential mass spectrum

$$
\rho(m) \sim \frac{C}{m^{a}} \mathrm{e}^{m / T_{0}},
$$

with $T_{0}$ of order $m_{\pi}$. While the small variation of $T_{0} \approx m_{\pi}$ from version to version is of no physical importance, the nature of the system when $T \rightarrow T_{0}$ depends critically on the power $a$ of $m$ in Eq. (23.44). We now study this in order to determine how the behavior of $\rho$ determines the physical properties of fireballs.

Inserting $1=\int \delta_{0}\left(m^{2}-p^{2}\right) \mathrm{d} m^{2}$ and replacing $\tau\left(m^{2}\right) \mathrm{d} m^{2}$ by $\rho(m) \mathrm{d} m$ in Eq. (23.41), we find

$$
Z_{1}(\beta, V)=\frac{V^{\mathrm{ex}} T}{2 \pi^{2}} \int m^{2} \rho(m) K_{2}(m \beta) \mathrm{d} m .
$$

As we are interested in the behavior at $T \rightarrow T_{0}\left(\beta \rightarrow \beta_{0}\right)$, we denote all quantities which are constant in this limit by the symbol $C$ (at each place where it occurs, $C$ may have a different value and/or dimension). Using Eq. (23.44) and the asymptotic formula $K_{2}(x) \sim \sqrt{\pi / 2 x} \mathrm{e}^{-x}$, we obtain

$$
Z_{1}(\beta, V) \underset{T \rightarrow T_{0}}{\sim} C \int_{M}^{\infty} m^{3 / 2-a} \mathrm{e}^{-\left(\beta-\beta_{0}\right) m} \mathrm{~d} m+C .
$$

Here $M$ is a mass large enough to justify the use of the asymptotic form of $K_{2}$ and Eq. (23.44), while $+C$ stands for the non-singular integral from $m_{\pi}$ to $M$. With $\beta-\beta_{0} \sim C\left(T-T_{0}\right)=C \Delta T$, we find

$$
Z_{1}(\beta, V) \underset{T \rightarrow T_{0}}{\sim} \begin{cases}C+C \Delta T^{a-5 / 2}, & a \neq 5 / 2, \\ C-\ln \frac{\Delta T}{T_{0}}, & a=5 / 2 .\end{cases}
$$


Table 23.1 Thermodynamic quantities calculated from Eq. (23.47)

\begin{tabular}{l|l|l|l|l|l}
\hline$a$ & $P$ & $n$ & $\varepsilon$ & $\delta \varepsilon / \varepsilon$ & $C_{V}=\mathrm{d} \varepsilon / \mathrm{d} T$ \\
\hline $1 / 2$ & $C / \Delta T^{2}$ & $C / \Delta T^{2}$ & $C / \Delta T^{3}$ & $C+C \Delta T$ & $C / \Delta T^{4}$ \\
\hline 1 & $C / \Delta T^{3 / 2}$ & $C / \Delta T^{3 / 2}$ & $C / \Delta T^{5 / 2}$ & $C+C \Delta T^{3 / 4}$ & $C / \Delta T^{7 / 2}$ \\
\hline $3 / 2$ & $C / \Delta T$ & $C / \Delta T$ & $C / \Delta T^{2}$ & $C+C \Delta T^{1 / 2}$ & $C / \Delta T^{3}$ \\
\hline 2 & $C / \Delta T^{1 / 2}$ & $C / \Delta T^{1 / 2}$ & $C / \Delta T^{3 / 2}$ & $C+C \Delta T^{1 / 4}$ & $C / \Delta T^{5 / 2}$ \\
\hline $5 / 2$ & $C \ln \left(T_{0} / \Delta T\right)$ & $C \ln \left(T_{0} / \Delta T\right)$ & $C / \Delta T$ & $C$ & $C / \Delta T^{2}$ \\
\hline 3 & $P_{0}-C \Delta T^{1 / 2}$ & $n_{0}-C \Delta T^{1 / 2}$ & $C / \Delta T^{1 / 2}$ & $C / \Delta T^{1 / 4}$ & $C / \Delta T^{3 / 2}$ \\
\hline $7 / 2$ & $P_{0}-C \Delta T$ & $n_{0}-C \Delta T$ & $\varepsilon_{0}$ & $C / \Delta T^{1 / 2}$ & $C / \Delta T$ \\
\hline 4 & $P_{0}-C \Delta T^{3 / 2}$ & $n_{0}-C \Delta T^{3 / 2}$ & $\varepsilon_{0}-C \Delta T^{1 / 2}$ & $C / \Delta T^{3 / 4}$ & $C / \Delta T^{1 / 2}$ \\
\hline
\end{tabular}

In Table 23.1, we list the most interesting quantities for $a=1 / 2,2 / 2, \ldots, 8 / 2$, namely, pressure $P$, fireball number density $n$, energy density $\varepsilon$, mean relative fluctuations $\delta \varepsilon / \varepsilon$ of $\varepsilon$, and specific heat $C_{V}=\mathrm{d} \varepsilon / \mathrm{d} T$. We notice that, as $T \rightarrow T_{0}$ $(\Delta T \rightarrow 0$ ), the energy density diverges for $a<7 / 2$. Thus only for $a<7 / 2$ can we expect $T_{0}$ to be a maximum temperature. For all cases we find for the velocity of sound: $v_{s}^{2}:=d P / d \epsilon \propto \Delta T$.

\section{Thermodynamics of Clustered Matter}

Let us consider a cluster with baryonic number $b$ enclosed in an 'external' fourvolume $V_{\mu}^{\mathrm{ex}}$. Then the one-cluster partition function $Z_{1, b}(\beta, V, b)$ is given by Eq. (23.41), the only change being the dependence of the mass spectrum on the baryonic number $b$. When $n$ such clusters are present, but each with the same $b$, we find for the $n$ cluster function the usual expression (23.37). When clusters with different $b$ are present, then we have to compute the product of the different contributions. Assume that $l$ clusters are present. Then the sum over all possible partitions of $b$ nucleons into $l$ clusters gives us the partition function of $b$ baryons assembled into $l$ clusters:

$$
Z_{b}(\beta, V, b, l):=\sum_{\left\{n_{j}\right\}}^{(l ; b)} \prod_{j=-\infty}^{\infty} \frac{1}{n_{j} !} Z_{1, b}(\beta, V, j)^{n_{j}} .
$$

The sum is over all partitions of $b$ baryons into $l$ clusters, with $n_{j}$ being the number of clusters having baryon number $j$ : 


$$
n_{j} \in\left\{n_{j} \geq 0, \quad \sum_{j=-\infty}^{\infty} n_{j}=l, \quad \sum_{j=-\infty}^{\infty} j n_{j}=b\right\} .
$$

In order to obtain the partition function of an arbitrary number of clusters having together $b$ baryons, we have to compute in Eq. (23.48) the sum over all possible numbers of clusters $l$, since each such configuration is possible. This has the net effect that the restriction $\sum n_{n}=l$ is removed:

$$
Z_{b}(\beta, V, b)=\sum_{\left\{n_{j}\right\}} \delta_{k}\left(b-\sum_{j=-\infty}^{\infty} j n_{j}\right) \prod_{j=-\infty}^{\infty} \frac{1}{n_{j} !} Z_{1, b}(\beta, V, j)^{n_{j}}
$$

We have made the constraint on baryonic number explicit.

The grand canonical partition function $Z$ is the L-transform of $Z_{b}$ in Eq. (23.49):

$$
Z(\beta, V, b)=\sum_{b=-\infty}^{\infty} \lambda^{b} Z_{b}(\beta, V, b)
$$

It is straightforward to carry out the sum over $b$ when Eq. (23.49) is inserted into Eq. (23.50), and we obtain

$$
Z(\beta, V, b)=\sum_{\left\{n_{j}\right\}} \prod_{j=-\infty}^{\infty} \frac{1}{n_{j} !}\left[\lambda^{j} Z_{1, b}(\beta, V, j)\right]^{n_{j}}
$$

All values of $n_{j}$ are allowed and the set $\left\{n_{j} \geq 0\right\}$ depends on $j$ only through the fact that there are $j$ members of the set. Since all $j$ are permitted, the order in which the infinite sum and product are evaluated is irrelevant, provided that the sum converges. Under this assumption, we obtain

$$
\begin{aligned}
Z(\beta, V, b) & =\prod_{j=-\infty}^{\infty} \sum_{n=0}^{\infty} \frac{1}{n !}\left[\lambda^{j} Z_{1, b}(\beta, V, j)\right]^{n} \\
& =\exp \left[\sum_{j=-\infty}^{\infty} \lambda^{j} Z_{1, b}(\beta, V, j)\right]
\end{aligned}
$$

which very much resembles the results of Sects. 23.3 and 23.3 [see Eq. (23.38)]:

$$
\begin{aligned}
\ln Z(\beta, V, \lambda) & =Z_{1}(\beta, V, \lambda), \\
Z_{1}(\beta, V, \lambda) & =\sum_{j=-\infty}^{\infty} \lambda^{j} Z_{1, b}(\beta, V, j) .
\end{aligned}
$$


Note that the existence of $Z_{1}(\beta, V, \lambda)$, the one-cluster grand canonical partition function, is not assured. In fact, often only the canonical partition function $Z_{b}$ in Eq. (23.49) exists. When an analytical expression for $Z$ can be found, then we can recover the physically relevant quantity $Z_{b}$ by the inverse L-transform.

\section{Partition Function of Nuclear Matter}

Thus we see that we need only to compute the one-cluster grand canonical partition function $Z_{1}$ to determine the grand canonical partition function $Z$ in Eq. (23.52). This is an easy task-we recall the definition of the function $\Phi_{b}$ in Eq. (23.30) and find

$$
Z_{1, b}(\beta, V, \lambda)=-\frac{2 V^{\mathrm{ex}}}{B_{b}(2 \pi)^{3}} \frac{\partial}{\partial \beta} \Phi_{b}(\beta, b)
$$

in the common rest frame of the volume and the 'thermometer', in complete analogy to Eq. (23.42). Consequently,

$$
Z_{1}(\beta, V, \lambda)=-\frac{V}{(2 \pi)^{3}} \frac{\partial}{\partial \beta} \sum_{b=-\infty}^{\infty} \frac{\lambda^{b}}{B_{b}} \Phi_{b}(\beta, b) .
$$

Were it not for the $b$ dependence of the function $B_{b}$ [see Eq. (23.26)], $B_{b} \sim M_{b}^{2}$, we would already have the analogue of Eq. (23.42).

In order to proceed further, we have to make an assumption about the $b$ dependence of the cluster mass $M_{b}$. For the present, we choose to consider the case

$$
M_{b}=\left\{\begin{array}{l}
b m_{\mathrm{N}},|b| \geq 1 \\
m_{\pi}, \quad b=0
\end{array}\right.
$$

where $m_{\pi}$ and $m_{\mathrm{N}}$ are the pion and nucleon masses, respectively. Here we have assumed that the mass of a ground-state cluster is proportional to the baryonic number. We now find for the grand canonical partition function

$$
\begin{aligned}
\ln Z(\beta, V, \lambda) & =Z_{1}(\beta, V, \lambda) \\
& =-\frac{V}{\mathscr{A}} \frac{1}{m_{\pi}^{2}} \frac{\partial}{\partial \beta} \Phi_{b}(\beta, 0)-\frac{V}{\mathscr{A}} \frac{1}{m_{\mathrm{N}}^{2}} \frac{\partial}{\partial \beta} \sum_{b \neq 0} \frac{\lambda^{b}}{b^{2}} \Phi_{b}(\beta, b) .
\end{aligned}
$$

In order to sum the expression (23.56), we can generate $b^{-2}$ in the sum by a double integral over $\lambda$.

While we can sum the general formula (23.56), we will be interested here in properties of bulk nuclear matter: that is, the case when a certain number of nucleons 
is already present in a given volume. Unless $T \sim T_{0}$, we expect only moderate contributions from baryon-antibaryon pair production, since $m_{\mathrm{N}} \gg T_{0}$. Therefore we further simplify our model and neglect now antibaryon production. We can implement this by restricting $b$ to be positive in Eq. (23.56). We note that in doing so we allow uncompensated baryon production, which is, for $T \leq T_{0}$, a small effect, ${ }^{5}$ since $m_{\mathrm{N}} / T_{0} \gtrsim 7$.

The bootstrap equation is then as it was before, viz., (23.9), but the input term that describes only 'raw' pions and nucleons takes the form

$$
\varphi(\beta, \lambda)=\varphi_{\pi}(\beta)+\lambda \varphi_{\mathrm{N}}(\beta) .
$$

The sum in Eq. (23.56) can now be obtained by integrating from zero to $\lambda$ :

$$
\begin{aligned}
-\frac{\mathscr{A}}{V} \ln Z(\beta, V, \lambda)= & \frac{1}{m_{\pi}^{2}} \frac{\partial}{\partial \beta} \Phi_{b}(\beta, 0) \\
& +\frac{1}{m_{\mathrm{N}}^{2}} \frac{\partial}{\partial \beta} \int_{0}^{\lambda} \frac{\mathrm{d} \lambda^{\prime}}{\lambda^{\prime}} \int_{0}^{\lambda^{\prime}} \frac{\mathrm{d} \lambda^{\prime \prime}}{\lambda^{\prime \prime}}\left[\Phi\left(\beta, \lambda^{\prime \prime}\right)-\Phi_{b}(\beta, 0)\right] .
\end{aligned}
$$

Given the grand canonical partition function $Z(\beta, V, \lambda)$, we want to obtain the quantities of physical interest for nuclear matter. The energy density, pressure, and baryon number density are, respectively,

$$
\begin{aligned}
\varepsilon(\beta, V, \lambda) & =-\frac{1}{V} \frac{\partial}{\partial \beta} \ln Z(\beta, V, \lambda), \\
P(\beta, V, \lambda) & =\frac{T}{V} \ln Z(\beta, V, \lambda), \\
\frac{\langle b\rangle}{V} & =: v(\beta, V, \lambda)=\frac{\lambda}{V} \frac{\lambda}{\partial \lambda} \ln Z(\beta, V, \lambda) .
\end{aligned}
$$

Of further physical interest is the energy per baryon $\varepsilon_{b}=\varepsilon / \nu$.

In the next section, we illustrate our model by some numerical results obtained by studying Eqs. (23.57)-(23.59).

\subsection{Properties of Nuclear Matter in the Bootstrap Model}

\section{The Different Phases}

In this section, we will study the physical properties of our model. We begin by considering in more detail the point $\varphi_{0}=\ln 4-1$, where the function $G(\varphi)$ [see

\footnotetext{
${ }^{5}$ However, we did find that as temperature rises this effect causes the rise of baryon density, a complete solution is presented in Chap. 27.
} 
Eq. (23.10)] has a square root singularity. This point corresponds to a curve $\lambda_{\mathrm{c}}=$ $f\left(\beta_{\mathrm{c}}\right)$ in the $(\lambda, \beta)$ plane, defined implicitly by the equation

$$
\varphi_{0}=\ln 4-1=\varphi_{\pi}\left(\beta_{\mathrm{c}}\right)+\lambda_{\mathrm{c}} \varphi_{\mathrm{N}}\left(\beta_{\mathrm{c}}\right) .
$$

Thus,

$$
\lambda_{\mathrm{c}}=\frac{\ln 4-1-\varphi_{\pi}\left(\beta_{\mathrm{c}}\right)}{\varphi_{\mathrm{N}}\left(\beta_{\mathrm{c}}\right)}, \quad \varphi_{i}(\beta)=\frac{\mathscr{A} m_{i}^{4}}{2 \pi^{2}} \frac{K_{1}\left(\beta m_{i}\right)}{\beta m_{i}}, \quad i=\pi, \mathrm{N} .
$$

We introduce the chemical potential $\mu$ by $\lambda=\mathrm{e}^{\beta \mu}$ and consider the function $\mu_{\mathrm{c}}=$ $f\left(T_{\mathrm{c}}\right)$, where $T_{\mathrm{c}}=\beta_{\mathrm{c}}^{-1}$, as follows from Eq. (23.60). As shown in Fig. 23.2, this line divides the $(\mu, T)$ plane into two parts. For $\mu<\mu_{\mathrm{c}}\left(T_{\mathrm{c}}\right)$, we have $\varphi<\varphi_{0}$ and we know that the grand canonical description is valid there. At $\varphi=\varphi_{0}$, we are on the critical curve corresponding to a singularity of $\ln Z$. We record the interesting behavior of $\mu_{\mathrm{c}}\left(T_{\mathrm{c}}\right)$ for small $T_{\mathrm{c}}$ (large $\beta_{\mathrm{c}}$ ). This can be found analytically, employing the asymptotic expansion for the Hankel functions; but we will not pursue this point here. From Fig. 23.2, we see that $\mu_{\mathrm{c}}$ increases initially as a function of $T_{\mathrm{c}}$. Beyond a certain point it drops continuously until $\mu_{\mathrm{c}}=0$ at $T_{\mathrm{c}}=T_{0}$. Here $\mathscr{A}$ was chosen to give $T_{0} \sim 150 \mathrm{MeV}$, which is a phenomenologically good value for a hadronic bootstrap. We note that the behavior of the chemical potential for $T \neq T_{0}$ is similar even when the pion term is switched off entirely (dashed line in Fig. 23.2).

The limiting temperature $T_{0}$ is now a solution of Eq. (23.60) with $\lambda_{\mathrm{c}}=1$. However, since the nuclear term is exponentially small at $\beta_{\mathrm{c}} \approx 1 / m_{\pi} \approx 1 / T_{0}$, we expect that the limiting temperature is but little changed from that of pionic bootstrap. The change of $T_{0}$ induced by the possible baryon production is obtained by expanding Eq. (23.60) around $\beta_{0}$. We find that the change of $T_{0}$ is negative: the limiting temperature is slightly lowered (by about $10 \mathrm{MeV}$ ) by the presence of nucleons.

There are three domains shown in Fig. 23.2. In domain I, enclosed by the function $\mu_{\mathrm{c}}\left(T_{\mathrm{c}}\right)$, the grand canonical description is valid; in domain II, above the critical curve, we have $\varphi>\ln 4-1$, but $T<T_{0}$. In this region, the description of physical quantities should be canonical, since the grand canonical partition function does not exist for $\varphi<\varphi_{0}$. It is possible, however, to consider the analytical continuation of the grand canonical function into this domain-inverse L-transform can then be used to find the canonical quantities. Henceforth, we will call region I the gaseous phase (because it contains the region of small density), and region II the 'liquid' phase (because it is approached if at fixed temperature the baryon density, i.e., $\lambda$ or $\mu$, increases). Region III, characterized by $T>T_{0}$, is a domain that cannot be reached from the physical phases in those bootstrap models that give divergent energy density at $T=T_{0}$. We have found, however, other versions of the nuclear bootstrap model which allow a transition even to this region-however, we will not discuss this possibility here.

We cannot exclude that, in models with more general input functions $\varphi$, a further phase develops for large baryon densities. However, this is not so within our simple 


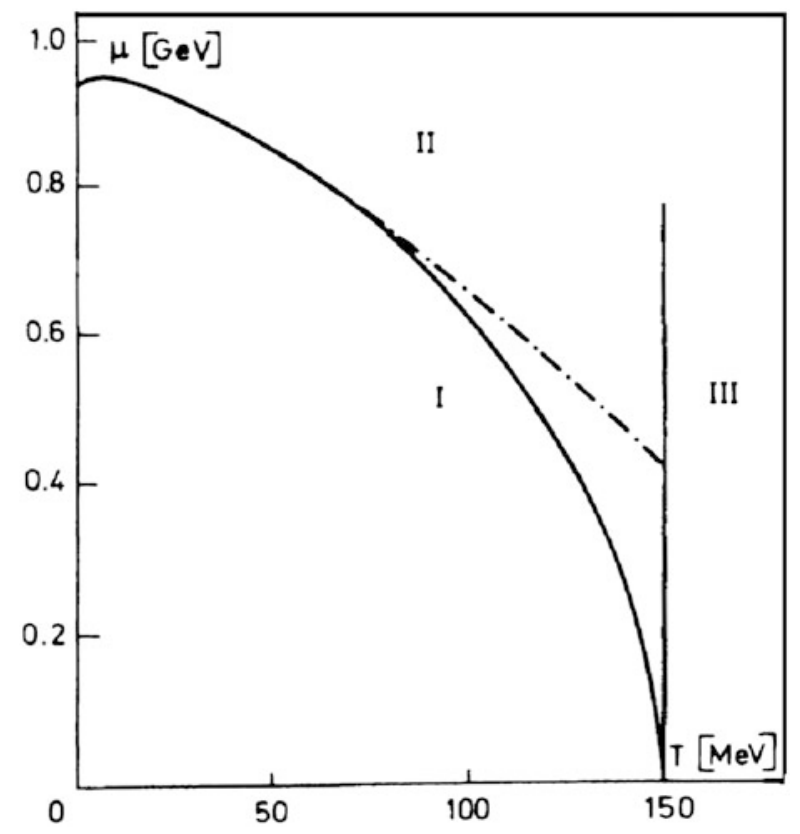

Fig. 23.2 The critical curve $\mu_{\mathrm{c}}=\mu_{\mathrm{c}}\left(T_{\mathrm{c}}\right)$ in the $(\mu, T)$ plane, separating the gaseous phase (I) from the 'liquid' phase (II). The dot-dashed line would be the critical curve if pions were excluded. Region III is inaccessible ( $T>T_{0}$ ): infinite energy density. For $T=0$, the critical chemical potential equals the nucleon mass; note that this is not its maximum value

model of pions and nucleons, where we neglect most of the details of nuclear structure. In particular, for $T \rightarrow 0$ and for $\mu$ corresponding to $v / \nu_{0} \sim 1$, we might need more detailed input than we have considered in the present simplified study.

\section{Baryon Density in the Gaseous Phase}

We begin with a short description of the numerical methods. We need to compute the different derivatives with respect to $\beta$ and $\lambda$ of Eq. (23.58). Since $\Phi(\beta, \lambda)=$ $G[\varphi(\beta, \lambda)]$, we need only to have the function $G(\varphi)$ and its derivatives with sufficient precision in order to calculate the quantities of physical interest. This is done by considering the expansion of $G(\varphi)$ at $\varphi_{0}$ :

$$
G(\varphi)=G_{0}-\left(\varphi_{0}-\varphi\right)^{1 / 2}-\frac{1}{6}\left(\varphi_{0}-\varphi\right)^{2 / 2}-\frac{1}{36}\left(\varphi_{0}-\varphi\right)^{3 / 2}+\Delta G(\varphi) .
$$

This equation defines the remainder $\Delta G$, which can be taken to have the polynomial form. Since we know the inverse function $\varphi=\varphi(G)$, we can easily fit $\Delta G$. We 
find that, even for a quite small degree of the polynomial $N(=3)$, already a very satisfactory result is obtained. This is partly due to the fact that Eq. (23.61) with $\Delta G$ neglected is, in itself, a very good approximation of $G$ since the maximum error occurs at $\varphi=0$ and is $\Delta G(\varphi)=5.7 \times 10^{-4}$. Also, at $\varphi=\varphi_{0}$, the proper analytic behavior is obtained from Eq. (23.61) for $G(\varphi)$ and its first and second derivatives. Thus to one per mile accuracy, the expansion (23.61) is already quite adequate; however, in numerical calculations, we have included the remainder $\Delta G$ in order to achieve a relative accuracy of $10^{-8}$. Another merit of the expansion (23.61) is its analytical integrability in Eq. (23.58). Thus we have succeeded in obtaining $\ln Z$ in terms of known functions. The computation of the different physical quantities, though tedious, is an elementary exercise now. The results were obtained and graphically processed by the CERN Interactive Computing System SIGMA [9]. An independent check of our calculations has been done with the Yellin expansion (23.13), wherever this was possible.

We begin the discussion of our results by considering the baryon number density $v$ [see Eq. (23.59c)] along the gas-'liquid' phase limit. As a unit of $V$, we will choose the 'elementary' volume of one baryon, $V_{\mathrm{N}}=m_{\mathrm{N}} A$, as introduced in Eq. (23.22), along with the constant $\mathscr{A}$ (not related to atomic and/or baryon number here denoted as $b$ ). The baryon number contained in the elementary volume $V_{\mathrm{N}}$ now follows from Eqs. (23.59c) and (23.58):

$$
V_{\mathrm{N}} v=-\frac{1}{m_{\mathrm{N}}} \frac{\partial}{\partial \beta} \int_{0}^{\lambda} \frac{\mathrm{d} \lambda^{\prime}}{\lambda^{\prime}}\left[G\left(\varphi_{\pi}+\lambda^{\prime} \varphi_{\mathrm{N}}\right)-G\left(\varphi_{\pi}\right)\right] .
$$

We find, upon differentiation,

$$
\begin{aligned}
V_{\mathrm{N}} v= & -\frac{1}{m_{\mathrm{N}}} \frac{\partial \varphi_{\pi}}{\partial \beta} \frac{\partial}{\partial \varphi_{\pi}} \int_{0}^{\lambda} \frac{\mathrm{d} \lambda^{\prime}}{\lambda^{\prime}}\left[G\left(\varphi_{\pi}+\lambda^{\prime} \varphi_{\mathrm{N}}\right)-G\left(\varphi_{\pi}\right)\right] \\
& -\frac{1}{m_{\mathrm{N}}} \frac{\partial \varphi_{\mathrm{N}}}{\partial \beta} \frac{1}{\varphi_{\mathrm{N}}}\left[G\left(\varphi_{\pi}+\lambda \varphi_{\mathrm{N}}\right)-G\left(\varphi_{\pi}\right)\right] .
\end{aligned}
$$

At the critical line, we just have $\varphi_{\pi}+\lambda \varphi_{\mathrm{N}}=\varphi_{0}$, so

$$
\begin{aligned}
\left.V_{\mathrm{N}} v\right|_{\text {crit }}= & -\left.\left[\ln 2-G\left(\varphi_{\pi}\right)\right] \frac{1}{m_{\pi}} \frac{\partial}{\partial \beta} \ln \varphi_{\mathrm{N}}\right|_{\text {crit }} \\
& -\left.\frac{1}{m_{\mathrm{N}}} \frac{\partial \varphi_{\pi}}{\partial \beta} \frac{\partial}{\partial \varphi_{\pi}} \int_{0}^{\lambda} \frac{\mathrm{d} \lambda^{\prime}}{\lambda^{\prime}}\left[G\left(\varphi_{\pi}+\lambda^{\prime} \varphi_{\mathrm{N}}\right)-G\left(\varphi_{\pi}\right)\right]\right|_{\text {crit }} .
\end{aligned}
$$

The first term is the only one remaining in the absence of pions and is shown as a dash-dotted line in Fig. 23.3. Since for $T \leq T_{0}$ we have $m_{\mathrm{N}} / T \gg 1$, the asymptotic form for the Bessel function in $\varphi_{\mathrm{N}}$ can be used to determine $v$. Therefore, we find 


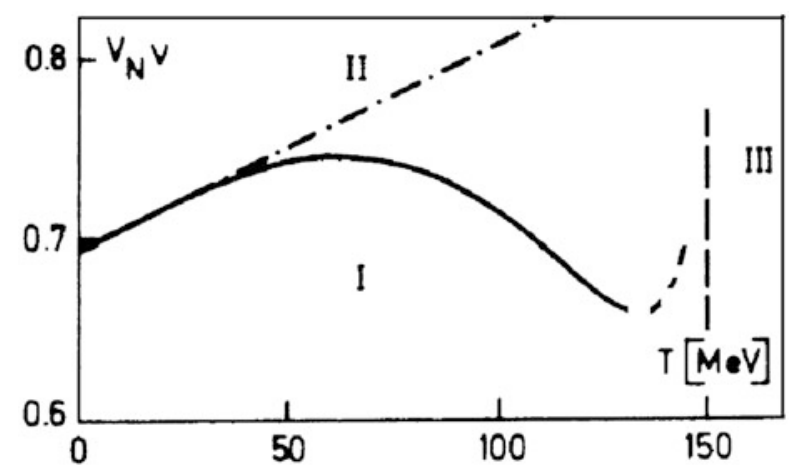

Fig. 23.3 Critical baryon number per nucleon volume $V_{\mathrm{N}}$ as a function of the temperature. The dot-dashed line results if pions are excluded. The unexpected shape of the critical curve is seen to be due to the coexistence of pions and nucleons. Region I is gaseous, while region II is fluid

$$
\left.V_{\mathrm{N}} v\right|_{\text {crit, no pions }}=\left(1+\frac{3 T}{2 m_{\mathrm{N}}}\right) \ln 2
$$

Even including pions, this expression is correct for low temperatures since, as

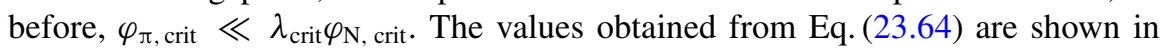
Fig. 23.3. We see that the onset of the pion component lowers the phase transition density, but at high temperatures, the density again increases sharply. ${ }^{6}$

We notice that, for $T<T_{0}-\delta$ (with $\delta$ a few $\mathrm{MeV}$ ), the transition from gaseous to 'liquid phases' occurs always below one (one baryon per unit volume is by definition the normal nuclear density). This justifies a posteriori our choice for the names of the different phases.

In Fig. 23.4, we show the baryon density in the gaseous phase: in Fig. 23.4a as a function of chemical potential with temperature being the parameter (isotherms), in Fig. $23.4 \mathrm{~b}$ as a function of temperature, with the chemical potential as a parameter. In Fig. 23.4c, d, we have eliminated the chemical potential from Fig. 23.4a and replaced it by the pressure [see Eq. (23.59b)] in units of $P_{0}=m_{\mathrm{N}} / V_{\mathrm{N}}=\mathscr{A}^{-1}$. In Fig. 23.4a, $\mu=0$ implies a finite baryon density, particularly noticeable for $T \gtrsim 120 \mathrm{MeV}^{7}$

\footnotetext{
${ }^{6}$ This mirrors the behavior of the rapidly changing factor $\exp [(m-\mu) / T]$; hadronic matter at phase boundary is meson dominated for $T>m_{\pi} / 2 \mathrm{MeV}$. Moreover, after we allowed for antimatter production (see solution presented in Chap. 27) the net baryon density continues to decrease for $T \rightarrow T_{0}$.
}

${ }^{7}$ This is another artifact of the approximation to ignore antibaryons. 

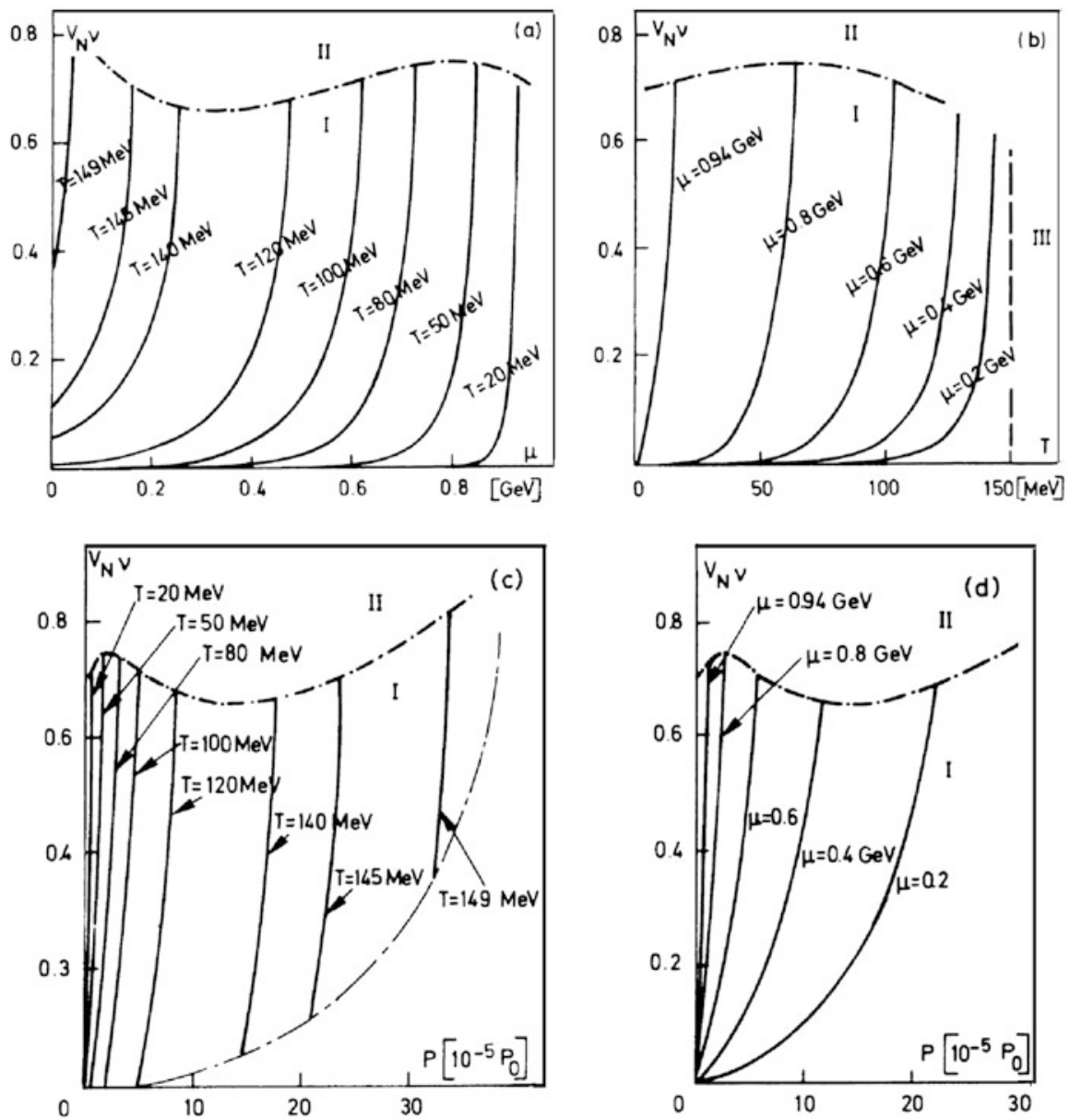

Fig. 23.4 Baryon number per nucleon volume $V_{\mathrm{N}}$ in various representations up to the critical curve: (a) against chemical potential with isotherms, (b) against the temperature with $\mu$ as parameter, (c) against the pressure with isotherms, (d) against the pressure with $\mu$ as parameter. $P_{0}=\mathscr{A}^{-1} \approx$ proton rest energy density ('internal proton pressure'). The dash-dotted curve is the critical curve, and region II the liquid phase. The white lower right corner in (c) is due to the impossibility of having antibaryons at high temperature (asymmetry of our input term)

\section{Baryon Energy in the Gaseous Phase}

The energy contained in the unit volume $V_{\mathrm{N}}$ can easily be obtained from Eqs. (23.59a) and (23.58):

$$
V_{\mathrm{N}} \varepsilon(\beta, \lambda)=\frac{m_{\mathrm{N}}}{m_{\pi}^{2}} \frac{\partial^{2}}{\partial \beta^{2}} G\left(\varphi_{\pi}\right)+\frac{1}{m_{\mathrm{N}}} \frac{\partial^{2}}{\partial \beta^{2}} \int_{0}^{\lambda} \frac{\mathrm{d} \lambda^{\prime}}{\lambda^{\prime}} \int_{0}^{\lambda^{\prime}} \frac{\mathrm{d} \lambda^{\prime \prime}}{\lambda^{\prime \prime}}\left[G\left(\varphi_{\pi}+\lambda^{\prime \prime} \varphi_{\mathrm{N}}\right)-G\left(\varphi_{\pi}\right)\right] .
$$


Fig. 23.5 Energy per nucleon volume $V_{\mathrm{N}}$ as a function of baryon number per nucleon volume.

Isotherms up to the critical curve separating gas (I) from liquid (II). As the rest mass is included in the energy per nucleon volume, the lower part of the diagram remains empty

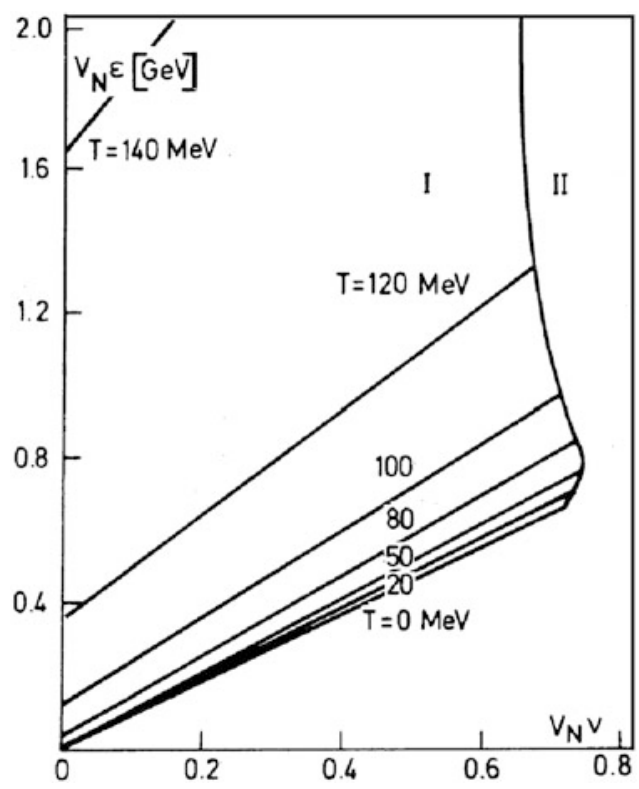

Both Eqs. (23.66) and (23.62) are functions of $\mu$ and $T$, and we can eliminate numerically either one of these physical parameters in Eq. (23.66) and replace it by $v$ [see Eq. (23.62)]. Since $T$ has a better intuitive meaning, we eliminate the chemical potential from Eq. (23.66) and consider

$$
\varepsilon(\beta, \lambda)=\varepsilon(\beta, \lambda(\beta, v))=\varepsilon_{v}(\beta, v)
$$

dropping henceforth the lower subscript $v$. The results are shown in Fig. 23.5. Here the isotherms $T=$ constant are shown for $V_{\mathrm{N}} \varepsilon$ as a function of $V_{\mathrm{N}} \nu$. We record the nearly linear behavior (in the gas phase) of the energy density: $\varepsilon \sim C_{1}+C_{2} v$, with temperature-dependent constants $C_{1}, C_{2}$. We recall that, for very small $v(T)$, our neglect of antibaryons is not justified. But above $V_{\mathrm{N}} v=0.1$ and $T \lesssim 120 \mathrm{MeV}$, our results should be independent of this approximation.

Even better insight can be obtained by inspecting the energy per baryon, excluding the rest mass,

$$
\mathscr{E}_{b} n r:=\frac{\varepsilon(\beta, v)}{v}-m_{\mathrm{N}}=\mathscr{E}_{b}-m_{\mathrm{N}}
$$

shown in Fig. 23.6. For small temperatures and densities, this should be just the usual $3 T / 2$, which we actually find for $T=20 \mathrm{MeV}$. For higher temperatures, as we can see in Fig. 23.6b, this is the lower limit of the thermal and interaction energy $\mathscr{E}_{b} n \mathrm{r}$. For $T=50 \mathrm{MeV}$ and higher, we have a large pion component; thus the energy per baryon (total energy divided by total baryon number), which also 

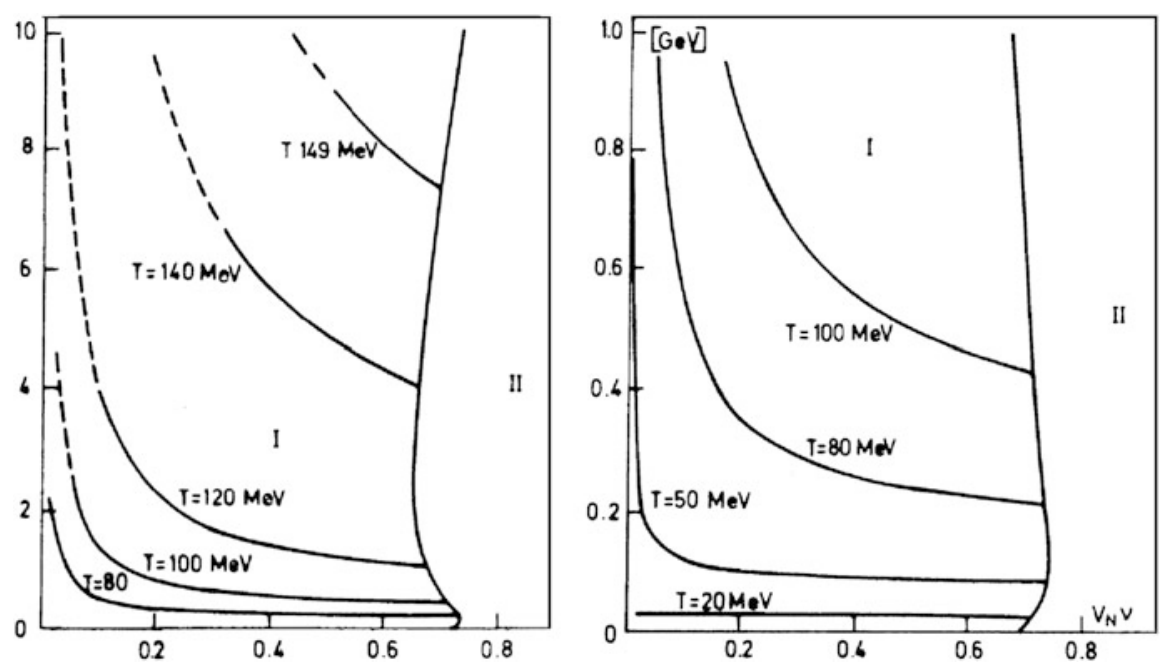

Fig. 23.6 Energy per baryon minus rest mass $\mathscr{E}_{b}^{n r}=E / b-m_{\mathrm{N}}$ as a function of baryon number per nucleon volume $V_{\mathrm{N}} v$ : (left: from 0 to $10 \mathrm{GeV}$; right: from 0 to $1 \mathrm{GeV}$ ) with isotherms up to the critical curve separating gas (I) from fluid (II)

includes the energy of the pions, stays high above the lower limit $3 T / 2$. We note that our interaction energy is, by definition, positive. Our nuclear mass $m_{\mathrm{N}}$ for the input nucleon should, in principle, include all the binding effects at saturation, and thus be really $m_{\mathrm{N}}-E_{\mathrm{B}}$. Therefore, at densities lower than the saturation density in the gaseous phase, the thermal energy $3 T / 2$ is the lower limit on the energy per baryon. Furthermore, we note that, within our model, the thermal energy dominates the picture between $\sim 20$ and $\sim 60 \mathrm{MeV}$, at which point the onset of pion and resonance excitation becomes important.

It is a straightforward matter to isolate the thermal term from Eq. (23.66). In fact, recalling the rules of chain differentiation, we obtain from Eqs. (23.59a), (23.59c), and (23.58),

$$
\varepsilon=-\frac{1}{V} \frac{\partial \varphi_{\pi}}{\partial \beta} \frac{\partial}{\partial \varphi_{\pi}} \ln Z-v \frac{\partial}{\partial \beta} \ln \varphi_{\mathrm{N}} .
$$

The first term expresses the pion-nucleon interacting component and, as discussed in Sect. 23.4, it is small at temperatures below $60 \mathrm{MeV}$. The second term is just the 'free' nucleon term at density $v$, which in the non-relativistic limit gives us the usual $3 T v / 2$. 


\subsection{Summary}

We have generalized the Statistical Bootstrap Model in a suitable way, which allows for the description of clustering hadron matter with constant energy density and a conserved quantum number. We apply our theory to the particular case of nuclear matter which, in the thermodynamic equilibrium, consists at finite temperature of nuclear clusters and their excitations, pions, and mesonic and baryonic resonances. Although in the general theoretical part of our work we have maintained baryon number conservation, in the numerical part, we study the properties of nuclear matter, neglecting the antibaryon production.

At many stages of our model, relatively arbitrary assumptions have been made, which can only be justified a posteriori by a comparison with the experimental results; or, perhaps, by the beauty and simplicity of the theoretical relation and intuition and experience. In particular:

1. we assume an ad hoc ansatz for the bootstrap equation for the nuclear level density [see Eq. (23.19)];

2. we assume a relation of $\sigma$ with the mass spectrum [see Eqs. (23.25) and (23.20)];

3. we take the proper volume as being parallel to the momentum of the fireball;

4. we assume that the natural volumes of the input particles grow with their mass.

As a consequence of (iii) and (iv), we have found that the energy density of fireballs is constant [see Eq. (23.22)] and equal to $\mathscr{A}^{-1}$, which is the only arbitrary parameter of our model. Although our calculations are more in the nature of an exploratory study than a final result, we believe that some of the general features we find in our model are relatively model-independent and could survive further elaboration.

1. Considering the grand canonical partition function, depending on the chemical potential and temperature, we find three different situations:

(i) a gaseous state (containing the empty vacuum for $\mu \rightarrow 0, T=0$ ), characterized by the presence of easily movable but strongly interacting nuclei and pions, all in arbitrary states of excitation;

(ii) a 'liquid' phase at larger baryon densities; and

(iii) a supercritical (unphysical) region above $T=T_{0}=150 \mathrm{MeV}$, where the energy density becomes infinite.

2. The transition to the 'liquid' phase occurs at about $0.65-0.75$ of the normal nuclear number density and at finite energy density, except when $T$ approaches $T_{0}$, where the pure gaseous phase persists through high density and where the energy density becomes very large. We would like to mention now that what we have called throughout this paper the 'liquid' phase is really the coexistence of two phases, vapor and liquid, in equilibrium. We are currently working on a description of the high density region beyond the phase transition from gas to liquid.

3. In our actual description, we find a limiting temperature $T_{0} \approx 150 \mathrm{MeV}$. At this temperature, the energy density diverges. We have noted, however, that this is a 
subtle point which touches on the limits of validity of our present interpretation of the mass spectrum. In this respect, we recall that the volume of fireballs now grows with the fireball mass - thus the average density should be finite for $T \rightarrow$ $T_{0}$. In a consistent model, we expect a finite energy density at $T_{0}$, so that the presently forbidden region beyond $T_{0}$ will now become accessible.

4. Below $60 \mathrm{MeV}$, we find that the energy per baryon obeys roughly the simple relation $\sim 3 T / 2$; however, below $20 \mathrm{MeV}$, our model includes too little nuclear structure to have enough predictive power. Above $60 \mathrm{MeV}$, we find that pion degrees of freedom absorb an increasing amount of the total energy, so that the 'energy per baryon' (the total energy/number of baryons) exceeds more and more the energy which the baryons themselves carry.

Looking ahead, we hope to enlarge our model by making the input more elaborate, by maintaining the particle-antiparticle symmetry, and by considering the particular importance of alpha clusters. It seems that a profound study of the 'liquid' phase will be rewarding since much of the structure of the liquid (maybe even the existence of a new 'solid' phase) depends on the amount of nucleon structure we include in the input terms. An obvious first step in this direction is the possible introduction of effective masses ( $<$ free masses) of the bound nucleons, a feature that is very likely relevant to the understanding of the saturation of nuclear matter in the bootstrap description. We must also incorporate Fermi and Bose statistics and investigate models leading to a finite energy density at $T_{0}$.

Open Access This book is distributed under the terms of the Creative Commons Attribution Noncommercial License which permits any noncommercial use, distribution, and reproduction in any medium, provided the original author(s) and sources are credited.

\section{References}

1. R. Hagedorn, J. Rafelski, Phys. Lett. B 97, 136 (1980)

2. R. Hagedorn, I. Montvay, J. Rafelski, Thermodynamics of nuclear matter from statistical bootstrap model. Preprint CERN-TH-2605 (1979); In Hadronic Matter at Extreme Energy Density, ed. by N. Cabibbo, L. Sertorio (Plenum Press, New York, 1980), pp. 49-148

3. H. Koppe, Phys. Rev. 76, 688 (1949)

4. E. Fermi, Prog. Theor. Phys. 5, 570 (1950)

5. S.Z. Belenkij, Nucl. Phys. 2, 259 (1956)

6. Particle Data Group, Phys. Lett. 75B, 1 (1978)

7. J. Yellin, Nucl. Phys. B 52, 583 (1973)

8. W. Nahm, Nucl. Phys. B 45, 525 (1972)

9. R. Hagedorn, J. Reinfelds, C. Vandoni, L. Van Hove, SIGMA, A New Language for Interactive Array-Oriented Computing. Yellow Report CERN-78-12 (1978) 